\title{
Communication
}

\section{Evidence for essential histidine and dicarboxylic amino-acid residues in the active site of UDP-glucose : solasodine glucosyltransferase from eggplant leaves}

\author{
Paulina Nawłoka, Małgorzata Kalinowska, Cezary Pączkowski and \\ Zdzisław A. Wojciechowski ${ }^{\varpi}$ \\ Institute of Biochemistry, Warsaw University, Warszawa, Poland \\ Received: 08 April, 2003; revised: 27 May, 2003; accepted: 02 June, 2002
}

Key words: glycoalkaloids, solasodine, glucosyltransferase, active site, Solanum melongena

\begin{abstract}
Effects of several chemical probes selectively modifying various amino-acid residues on the activity of UDP-glucose : solasodine glucosyltransferase from eggplant leaves was studied. It was shown that diethylpyrocarbonate (DEPC), a specific modifier of histidine residues, was strongly inhibitory. However, in the presence of excessive amounts of the enzyme substrates, i.e. either UDP-glucose or solasodine, the inhibitory effect of DEPC was much weaker indicating that histidine (or histidines) are present in the active site of the enzyme. Our results suggest also that unmodified residues of glutamic (or aspartic) acid, lysine, cysteine, tyrosine and tryptophan are necessary for full activity of the enzyme. Reagents modifying serine and arginine residues have no effect on the enzyme activity.
\end{abstract}

Steroidal glycoalkaloids occur in numerous species of the Solanaceae including such economically important crop plants as potato (Solanum tuberosum), tomato (Lycopersicon esculentum) or garden eggplant (Solanum melongena). For a long time these glycosidic compounds have attracted much attention due to their toxicity for animals and humans, interesting pharmacological activities of some of them and their physiological function in

\footnotetext{
This work was supported in part by research grant BW 1485/43/2000 from the Faculty of Biology, Warsaw University awarded to C.P.

${ }^{\circledR}$ To whom correspondence should be addressed: Zdzisław A. Wojciechowski, Instytut Biochemii UW, I. Miecznikowa 1, 02-096 Warszawa, Poland; phone: (48 22) 554 3305, fax: (48 22) 5543221 e-mail: zawoj@biol.uw.edu.pl
}

Abbreviations: GlcTase, UDPGlc: solasonide glucosyltransferase; other abbreviations are given in Materials and Methods. 
plants as resistance factors against pathogenic fungi, nematodes, molluscs and insects (Friedman \& McDonald, 1999; Roddick, 1996; Valkonen et al., 1996). In spite of numerous studies concerning the occurrence, chemical structure and biological activities of steroidal glycoalkaloids little has been known until recently about the biosynthesis of their aglycones and, particularly, about the formation of the sugar chains present in these compounds. In the last decade it has been reported that potato (Zimowski, 1991; Stapleton et al., 1991; Bergenstrahle et al., 1992), tomato (Zimowski, 1994) and eggplant (Pączkowski \& Wojciechowski, 1994; Pączkowski et al., 1997) contain specific glycosyltransferases catalyzing glucosylation or galactosylation of steroidal alkaloids typical for these plants, i.e. solanidine, tomatidine and solasodine, respectively. These UDPGlcor UDPGal-dependent enzymes, most probably catalyzing the first step in the formation of the sugar chains present in glycoalkaloids characteristic for the above-mentioned plants, have been at least partially purified and characterized (Stapleton et al., 1992; Zimowski, 1998; Paczkowski et al. 1997; 1998; 2001). However, up to date, there are no informations about the mechanisms of the reactions catalyzed by these glycosyltransferases.

The present study was a continuation of our previous investigations on UDPGlc : solasodine glucosyltransferase (GlcTase) from eggplant leaves. The effects of a number of group-specific chemical probes on the activity of eggplant GlcTase were investigated in order to elucidate the involvement of specific amino-acid residues in the active site of this enzyme.

\section{MATERIALS AND METHODS}

Group-specific chemical probes: diethylpyrocarbonate (DEPC), p-chloromercuribenzenesulfonic acid (PCMBS), 4,4'-diisothiocyanatostilbene-2,2'-disulfonic acid (DIDS), 4-aceta- mido-4'-isothiocyanatostilbene-2,2' -disulfonic acid (SITS), pyridoxal 5'-phosphate (PLP), phenylglyoxal (PG), phenylmethylsulfonyl fluoride (PMSF), $N$-acetylimidazole (NAI), 2-hydroxy-5-nitrobenzyl bromide (HNB, Koshland I Reagent), 2-mercaptoethanol (2-ME), dithiothreitol (DTT) and dithioerythritol (DTE) were acquired from Sigma. Woodward's Reagent K (WRK) and imidazole were obtained from Fluka. Commercial preparation of solasodine (from Sigma) was additionally purified by recrystallization from ethanol immediately before use. Tritium-labelled tigogenin was synthesized as described earlier (Pączkowski et al., 2001).

Enzyme preparations. Leaves of 9-week-old eggplants (Solanum melongena L.) were used for enzyme isolation as described previously (Paczkowski \& Wojciechowski, 1994). Further purification of GlcTase was achieved as described in our preceding paper (Pączkowski et al., 1997).

Standard GlcTase assays. Unless stated otherwise, incubations were carried out at $30^{\circ}$ for $30 \mathrm{~min}$. In a typical enzyme assay $0.50 \mathrm{ml}$ of the enzyme ( $30 \mu \mathrm{g}$ protein) in $0.05 \mathrm{M}$ Hepes buffer, $\mathrm{pH}$ 7.3, was preincubated with an amino acid-specific probe in $0.01-0.10 \mathrm{ml}$ of water (DTE, DTT, HNB, 2-ME, PCMBS, PG, WRK) or in $0.01-0.10 \mathrm{ml}$ of $0.05 \mathrm{M}$ Hepes, pH 7.3 (DIDS, SITS, NAI, PLP, PMSF) or in $0.01 \mathrm{ml}$ of ethanol (DEPC). Control samples contained, instead of chemical probe solutions, equivalent amounts of pure solvents. Unless stated otherwise, the enzyme reaction was initiated by the addition of UDP- $\left[{ }^{3} \mathrm{H}\right] \mathrm{Glc}$ (1.5 nmol, $3.33 \mathrm{kBq})$ or UDP-[ $\left.{ }^{14} \mathrm{C}\right] \mathrm{Glc}(6.8$ pmol, $3.33 \mathrm{kBq}$ ) in $0.01 \mathrm{ml}$ of $50 \%$ ethanol and solasodine $(0.4 \mathrm{nmol})$ in $0.01 \mathrm{ml}$ of ethanol. Incubations were carried out at $30^{\circ}$, usually for $30 \mathrm{~min}$. The reaction was stopped by heating of the incubated samples in a boilling water-bath, the labelled glucosidic product was separated from the labelled nucleotide sugar precursor by extraction with 1-butanol and quantitatively assayed as previously described (Pączkowski et al., 1998). 


\section{RESULTS AND DISCUSSION}

Selected data on the effects of amino acid modifying reagents on the activity of $S$. melongena GlcTase are given in Table 1. Significant inhibition was obtained in the presence of reagents which are known to react with $\varepsilon-\mathrm{NH}_{2}$ groups of lysine (DIDS, SITS and PLP), with histidine (DEPC), dicarboxylic quirement for reduced cysteine residues in the enzyme for its full activity. On the other hand, chemical probes reacting with arginine or serine, i.e. PG and PMSF, respectively, had no effect on GlcTase suggesting that these two amino acids are not essential for the activity of this enzyme.

In order to identify the amino acids involved in substrate binding, experiments were car-

Table 1. Effects of various chemical treatments on the activity of eggplant UDPGlc : solasodine GlcTase

\begin{tabular}{lcccc}
\hline Chemical probe* & $\begin{array}{c}\text { Concentration } \\
(\mathrm{mM})\end{array}$ & $\begin{array}{c}\text { Modified amino-acid } \\
\text { residue }\end{array}$ & $\begin{array}{c}\text { Relative activity** } \\
(\%)\end{array}$ & $\begin{array}{c}\mathrm{I}_{50}{ }^{* * *} \\
(\mathrm{mM})\end{array}$ \\
\hline & & & & \\
DIDS & 0.05 & Lys & 17 & 0.023 \\
SITS & 0.50 & Lys & 14 & 0.067 \\
PLP & 2.50 & Lys & 20 & 0.580 \\
DEPC & 0.30 & His & 11 & 0.081 \\
PCMBS & 0.10 & Cys & 13 & 0.018 \\
WRK & 0.07 & Asp, Glu & 8 & 0.028 \\
HNB & 4.0 & Trp & 20 & 2.60 \\
NAI & 17.0 & Tyr & 26 & 13.40 \\
PG & 5.0 & Arg & 107 & \\
PMSF & 0.03 & Ser & 99 & \\
$2-M E$ & 8.0 & Cys-Cys & 92 & \\
DTT & 8.0 & Cys-Cys & 96 & \\
DTE & 8.0 & Cys-Cys & & \\
\hline & & & & \\
\hline
\end{tabular}

\footnotetext{
*Abbreviations are explained in Materials and Methods; **enzyme activity in the absence of any chemical probe $=100 \%$; ****for strong inhibitors concentrations causing $50 \%$ inhibition are given.
}

amino-acids (WRK), tryptophan (HNB), tyrosine (NAI) and with free thiol groups in cysteine (PCMBS) indicating that the above listed amino acids are essential for full activity of the enzyme. It is noteworthy that the very strong inhibition of the enzyme by 0.07 mM PCMBS could be almost completely reversed by addition of $16 \mathrm{mM}$ 2-ME, DTT or DTE. This observation clearly confirms the re- ried out in which inhibition of GlcTase by various chemical probes was assayed after preincubation of the enzyme preparation with its substrate, i.e. UDPGlc or solasodine.

Very potent protection of the enzyme against inactivation with DEPC was found when enzyme preparation was preincubated with solasodine (see Fig. 1), suggesting that a histidine residue (or residues) is involved in 
the steroid substrate binding. Much smaller but significant protection of the enzyme by preincubation with solasodine was also observed using PCMBS, HNB and NAI, i.e. reagents modifying cysteine, tryptophan or tyrosine residues. Preincubation with sola-
Further experiments showed that the strong inhibition of GlcTase by $0.25 \mathrm{mM}$ DEPC could also be partially eliminated when the enzyme was preincubated with UDPGlc before addition of DEPC and solasodine. Preincubation with UDPGlc also substantially diminished

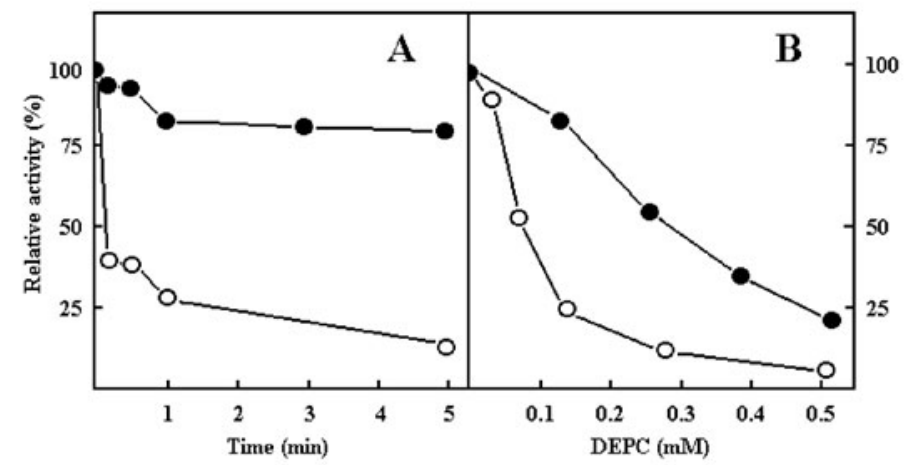

Figure 1. Protective effect of solasodine on inactivation of GlcTase by DEPC.

(A) The enzyme was preincubated for $5 \mathrm{~min}$ with (O) or without (O) $0.8 \mu \mathrm{M}$ solasodine then DEPC $(0.1 \mathrm{mM})$ was added and preincubation was continued. At various time intervals (0-5 min), $2 \mathrm{mM}$ histidine was added in order to destroy unreacted DEPC. The enzymatic reaction was initiated by addition of UDP- $\left[{ }^{14} \mathrm{C}\right] \mathrm{Glc}$. (B) After preincubation with or without solasodine, 0.1-0.5 mM DEPC was added and incubation continued for 5 min. Unreacted DEPC was destroyed with $2 \mathrm{mM}$ histidine and after further 5 min the enzymatic reaction was initiated with UDP-[ $\left.{ }^{14} \mathrm{C}\right] \mathrm{Glc}$.

sodine had no effect on the inhibition of the enzyme by other reagents tested. the inhibition by WRK, which is known to react with dicarboxylic amino acids, i.e. Asp or

Table 2. Protective effect of UDPGlc on the inhibitory effects of DEPC and WRK on solasodine glucosylation by eggplant GlcTase

Relative glucosylation rate (\%)

\begin{tabular}{|c|c|c|}
\hline Chemical probe & Preincubation & Preincubation \\
\hline
\end{tabular}

$\begin{array}{lcc}\text { None } & 100 & 100 \\ \text { DEPC }(0.25 \mathrm{mM}) & 15 & 29 \\ \text { WRK }(0.07 \mathrm{mM}) & 6 & 35\end{array}$

Enzyme preparation was preincubated for 5 min with or without UDP-[ $\left.{ }^{3} \mathrm{H}\right] \mathrm{Glc}$ (33.5 kBq, final conc. $\left.29 \mu \mathrm{M}\right)$. Then modifying reagent (DEPC or WRK) was added and preincubation was continued for 10 min. The enzymatic reaction was initiated by addition of unlabelled solasodine (final conc. $8 \mu \mathrm{M}$ ). In samples preincubated without UDPGlc the reaction was started using unlabelled solasodine and an equivalent amount of UDP- $\left[{ }^{3} \mathrm{H}\right] \mathrm{Glc}$. 
Glu (Table 2). The protection of the enzyme against inhibition by DEPC and WRK was particularly evident using another experimental approach (Table 3). In this case cold ing transfer of the sugar moiety from the nucleotide sugar donor to the acceptor (Kapitonov \& Yu, 1999). The results of our study strongly imply that histidine and dicar-

Table 3. Protective effect of UDPGlc on the inhibition by DEPC or WRK of $\left[{ }^{3} \mathrm{H}\right]$ tigogenin glucosylation by eggplant GlcTase

\begin{tabular}{lcc}
\hline & \multicolumn{2}{c}{ Relative glucosylation rate (\%) } \\
Chemical probe & Preincubation & Preincubation \\
& without UDPGlc & with UDPGlc \\
& & 100 \\
\hline None & 100 & 47 \\
DEPC $(0.30 \mathrm{mM})$ & 3 & 35 \\
WRK $(0.07 \mathrm{mM})$ & 8 & \\
\hline
\end{tabular}

The enzyme preparation was preincubated for $5 \mathrm{~min}$ with or without $29 \mu \mathrm{M}$ unlabelled UDPGlc. Then modifying reagent (DEPC or WRK) was added and preincubation was continued for another $10 \mathrm{~min}$. The enzymatic reaction was started by addition of $\left[{ }^{3} \mathrm{H}\right]$ tigogenin $(6.6 \mathrm{kBq}$, final conc. $10 \mu \mathrm{M})$.

UDPGlc was used for preincubation with the enzyme before addition of inhibitors and $\left[3-{ }^{3} \mathrm{H}\right]$ tigogenin as the steroid substrate. We have shown previously (Pączkowski et al., 2001) that tigogenin, a steroidal sapogenin which is a close structural analogue of solasodine, is a good substrate for the studied enzyme and, in contrast to solasodine, can be readily synthesized in a tritium-labelled form.

Genes for several plant glycosyltransferases catalyzing the nucleotide sugar-dependent glycosylation of various secondary plant metabolites, e.g. flavonols, anthocyanidins, limonoids, cyanohydrins or betanidins, have been cloned. A consensus sequence, so called PSPG-box, is present in all these enzymes and is considered to represent the nucleotide sugar binding site (Vogt \& Jones, 2000). The PSPG-box contains the highly conserved histidine and glutamic acid residues which are proposed to be involved in the nucleophilic $\mathrm{S}_{\mathrm{N}} 2$ reaction mechanism leading to the inversion of the anomeric sugar configuration dur- boxylic acid residues are essential for catalysis of glucosylation of steroidal alkaloids and sapogenins by the enzyme from $S$. melongena. They also indicate that a histidine residue(s) is important both for the binding of sugar nucleotide, i.e. UDP-glucose, as well as for the binding of steroidal aglycone. Moreover, our results suggest the importance of a glutamic (or aspartic) acid residue(s) for the binding of the steroid substrate.

\section{R E F E R E N C E S}

Bergenstrahle E, Tillberg E, Jonsson L. (1992) Plant Sci.; 84: 35-44.

Friedman M, McDonald GM. (1999) In Naturally occuring glycosides. Ikan $\mathrm{R}$, ed. pp 311-43. Wiley \& Sons, New York.

Kapitonov D, Yu RK. (1999) Glycobiology.; 9: 961-78. 
Pączkowski C, Kalinowska M, Wojciechowski ZA. (1997) Acta Biochim Polon.; 44: 43-54.

Pączkowski C, Kalinowska M, Wojciechowski ZA. (1998) Phytochemistry.; 48: 1151-9.

Pączkowski C, Kalinowska M, Wojciechowski ZA. (2001) Phytochemistry.; 58: 663-9.

Pączkowski C, Wojciechowski ZA. (1994) Phytochemistry.; 35: 1429-34.

Roddick JG. (1996) Adv Exp Med Biol.; 404: 277-95.

Stapleton A, Allen PV, Friedman M, Belknap WR. (1991) J Agric Food Chem.; 39: 1187-93.
Stapleton A, Allen PV, Tao HP, Belknap WR, Friedman M. (1992) Protein Expr Purif;; 3: 85-92.

Valkonen JPT, Keskitalo M, Vasara T, Pietila L. (1996) Crit Rev Plant Sci.; 15: 1-20.

Vogt T, Jones P. (2000) Trends Plant Sci.; 5: $380-6$.

Zimowski J. (1998) Plant Sci.; 136: 139-48.

Zimowski J. (1991) Phytochemistry.; 30: 1827-31.

Zimowski J. (1994) Acta Biochim Polon.; 41: 201-4. 